\title{
Helium Implantation Studies Utilizing the HIM. Turning a Bug into a Feature
}

Peter Hosemann ${ }^{1}$, Andrew Scott ${ }^{1}$, Sarah Stevenson ${ }^{1}$, Mehdi Balooch ${ }^{1}$, Zeeshan Mughal ${ }^{2}$, Frances Allen ${ }^{1}$, Yang Yang ${ }^{3}$, David Frazer ${ }^{4}$ and Marco Sebastiani ${ }^{2}$

${ }^{1}$ University of California Berkeley, Berkeley, California, United States, ${ }^{2}$ Roma Tre University, Rom, Lombardia, Italy, ${ }^{3}$ Lawrence Berkeley National Laboratory, Berkeley, California, United States, ${ }^{4}$ Los Alamos National Laboratory, Los Alamos, New Mexico, United States

Helium Ion beam Microscopy (HIM) is an exciting tool to image nonconductive and conductive samples at high resolution with unprecedented surface resolution. It has previously been used for very fine ion milling, orientation mapping, and/or imaging. Recently, more advanced technologies have been deployed in the systems such as TOF-SIMS [1]. While the tool has clear benefits, it remains an ion beam and therefore can also modify the samples investigated. Traditionally, this effect is not desirable but can be used in a beneficial fashion. Ion beam irradiations are widely conducted to understand a materials property change in nuclear environments. Helium especially is of interest since it is created through nuclear reactions in a material or through plasma surface interactions. Regardless the application, understanding how Helium, a Noble and insoluble gas, behaves in materials is of scientific interest to the material science community.

Helium is an insoluble gas known to create nanobubbles in materials leading to phenomena such as swelling or embrittlement. Furthermore, the bubbles can arrange themselves in an ordered fashion also known as Helium bubble superlattices [2]. Near-surface Helium implantation can also lead to blister in the material underneath the surface and delamination. Of course, these phenomena are detrimental to a materials performance and must be investigated and understood. In real nuclear applications the Helium bubble buildup can lead to component failures $[3,4]$.

In this work, we utilize the HIM to cause near-surface Helium damage and implant the Helium within the first $100 \mathrm{~nm}-300 \mathrm{~nm}$ at different dose and dose rates in single crystal and polycrystalline materials. We observe homogeneous swelling using Atomic Force Microscopy (Figure 1) and thermal property changes using thermal probes in an AFM [5]. It is found that the swelling relates to the Helium dose in a near linear fashion, regardless of the target material (W, V, Cu, Ti),. Additionally, we find that almost all materials tested create a Helium bubble superlattice up to specific dose threshold at which the bubbles are disarranged.

Detailed investigations are featuring the phenomenon of blistering and materials delamination. Again, AFM shows the onset of blistering, and TEM shows the reasons behind it. It is found that nanoscale bubbles coalescent and form larger cracks which in turn lead to surface delamination. Interestingly, the blisters appear to be faceted on single crystal materials (e.g. Tungsten shown in figure 2) and ion beam channeling can be observed on the blisters in TEM and in the HIM itself.

This work also features a series of HIM experiments in pre-and post FIBed structures to investigate the effect of free surfaces and stress constraints. It was found that the ring-core FIB-DIC method [4] makes the surface stress quantifiable and can be a driver for blistering. Residual stresses in the order of yield stress are reported using this method. However, swelling seems to be unaffected by external constraints and a simple stress model considering only the constraint of the surface seems inadequate to reveal the mechanism. 
In summary, this presentation will feature HIM, TEM, AFM, and FIB based techniques to allow a rapid throughput method to quantify Helium induced damage in materials while also studying the underlying mechanism of blistering at high doses.
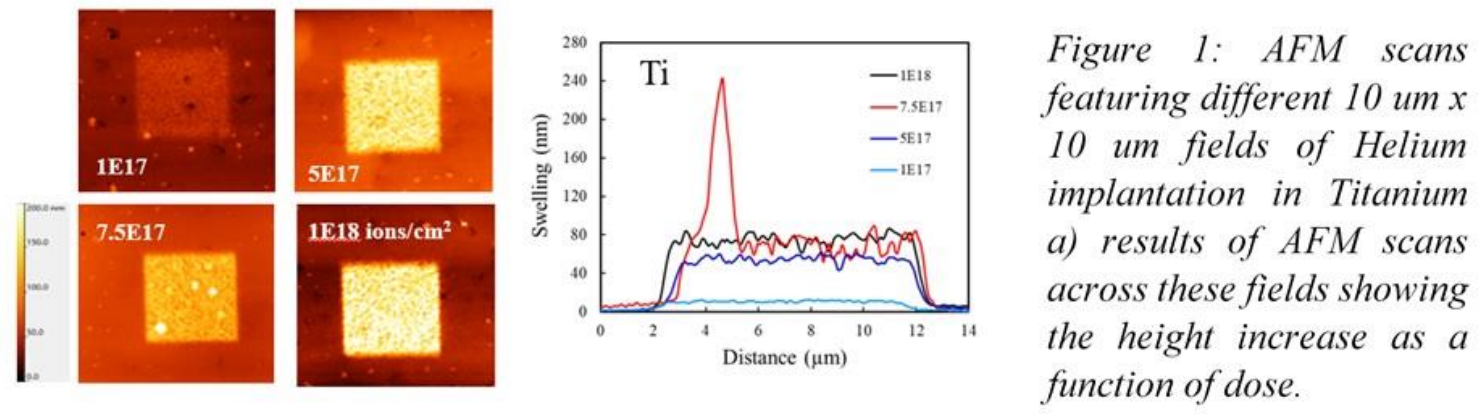

Figure 1. AFM scans featuring different 10 um x 10 um fields of Helium implantation in Titanium a) results of AFM scans across these fields showing the height increase as a function of dose.
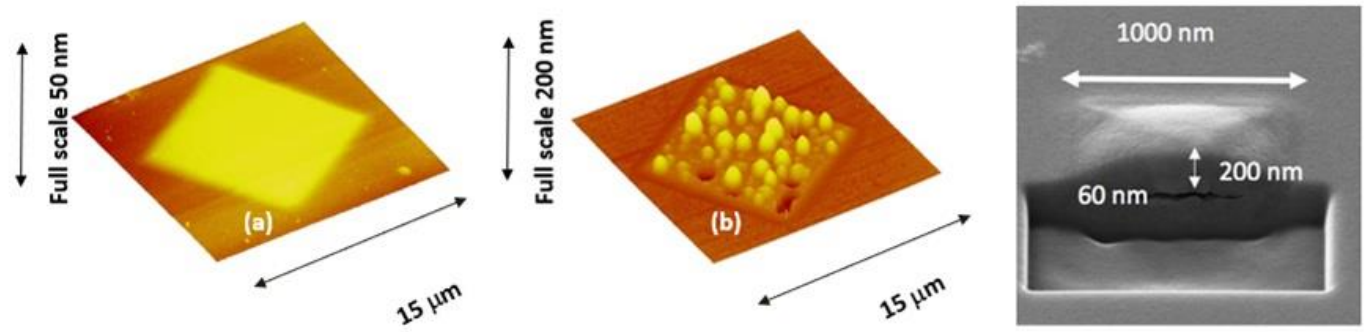

Figure 2: Swelling and blistering observed Tungsten via AFM and FIB cross section of blister formed on the surface.

Figure 2. Swelling and blistering observed Tungsten via AFM and FIB cross section of blister formed on the surface.

References

[1] N. Klingner, G. Hlawacek, R. Heller, J. von Borany, S. Facsko Micr. And Microanalysis 24 (S1) 802803 [2] Z.-J. Wang, F.I, Allen, Z.W.Shan, P. Hosemann, Acta Mat 121 (2016) 78-84

[2] F.I.Allen, P. Hosemann, M. Balooch, Scripta Mat $178 \quad$ (2020) 256260

[3] C.D. Judge S.Y. Persaud, A. Korinek, M.D. Wright Proceedings of the 18th International Conference on Environmental Degradation of Materials in Nuclear Power Systems - Water Reactors. The Minerals, Metals \& Materials Series. Springer, Cham https://doi.org/10.1007/978-3-030-04639-2_76[5] C. Howard, V. Bhakhri, C. Dixon, H. Rajakumar, C. Mayhew, C.D. Judge, J. NUcl Mat. 517 (2019) 17-34

[4] X. Song, K.B. Yeap, J. Zhu, J. Belnoue, M. Sebastiani, E. Bemporad, K.Y.Zeng, A. M. Korsunsky, Procedia Engineering 10 (2011) 2190-2195 